\title{
NF-KB2 signalling in enteroids modulates enterocyte responses to secreted factors from bone marrow-derived dendritic cells
}

Lauren G. Jones ${ }^{1}$, Andra Vaida', Louise M. Thompson', Felix I. Ikuomola', Jorge H. Caamaño $\mathbb{D}^{2}$, Michael D. Burkitt ${ }^{1}$, Fabio Miyajima ${ }^{3,4}$, Jonathan M. Williams ${ }^{5}$, Barry J. Campbell (1) ${ }^{1}$, D. Mark Pritchard ${ }^{1}$ and Carrie A. Duckworth (1) ${ }^{1}$

\begin{abstract}
Alternative pathway NF-KB signalling regulates susceptibility towards developing inflammatory bowel disease (IBD), colitis-associated cancer and sepsis-associated intestinal epithelial cell apoptosis and shedding. However, the cell populations responsible for the perturbed alternative pathway NF-KB signalling in intestinal mucosal pathology remain unclear. In order to investigate the contribution of the epithelial compartment, we have tested whether NF-kB2 regulated transcription in intestinal epithelial cells controls the intestinal epithelial response to cytokines that are known to disrupt intestinal barrier permeability. Enteroids were generated from the proximal, middle and distal regions of small intestine (SI) from C57BL/6J wild-type mice and displayed region-specific morphology that was maintained during sub-culture. Enteroids treated with $100 \mathrm{ng} / \mathrm{mL}$ TNF were compared with corresponding regions of SI from C57BL/6J mice treated systemically with $0.33 \mathrm{mg} / \mathrm{kg}$ TNF for $1.5 \mathrm{~h}$. TNF-induced apoptosis in all regions of the intestine in vitro and in vivo but resulted in Paneth cell degranulation only in proximal tissue-derived $\mathrm{Sl}$ and enteroids. TNF also resulted in increased enteroid sphericity (quantified as circularity from two-dimensional bright field images). This response was dose and time-dependent and correlated with active caspase-3 immunopositivity. Proximal tissuederived enteroids generated from $\mathrm{Nf}_{k} b 2^{-1-}$ mice showed a significantly blunted circularity response following the addition of TNF, IFNY, lipopolysaccharide (LPS) activated C57BL/6J-derived bone marrow-derived dendritic cells (BMDC) and secreted factors from LPS-activated BMDCs. However, NfKb $1^{-1-}$ mouse-derived enteroids showed no significant changes in response to these stimuli. In conclusion, the selection of SI region is important when designing enteroid studies as region-specific identity and response to stimuli such as TNF are maintained in culture. Intestinal epithelial cells are at least partially responsible for regulating their own fate by modulating NF-KB2 signalling in response to stimuli known to be involved in multiple intestinal and systemic diseases. Future studies are warranted to investigate the therapeutic potential of intestinal epithelial NF-KB2 inhibition.
\end{abstract}

\section{Introduction}

The intestine is lined by a single layer of columnar epithelial cells that function to maintain a barrier between

\footnotetext{
Correspondence: Carrie A. Duckworth (Carried@liverpool.ac.uk)

${ }^{1}$ Gastroenterology Research Unit, Department of Cellular and Molecular Physiology, Institute of Translational Medicine, University of Liverpool, Liverpool, UK

${ }^{2}$ Institute of Immunology and Immunotherapy, College of Medical and Dental Sciences, University of Birmingham, Birmingham, UK

Full list of author information is available at the end of the article.

Edited by A. Stephanou
}

luminal contents and the body ${ }^{1,2}$. Barrier function is maintained by the tight regulation of intestinal epithelial cell proliferation, apoptosis and cell shedding rates which are themselves modulated by several factors, including age, genetic background, dietary components, intestinal microbiota, enterally and parenterally administered drugs and other environmental factors ${ }^{3}$. Dysregulation of these cellular processes results in the break-down of the intestinal barrier and this is thought to be a contributing factor to the development of multiple diseases. We have

\section{(c) The Author(s) 2019}

(c) (i) Open Access This article is licensed under a Creative Commons Attribution 4.0 International License, which permits use, sharing, adaptation, distribution and reproduction cc) in any medium or format, as long as you give appropriate credit to the original author(s) and the source, provide a link to the Creative Commons license, and indicate if changes were made. The images or other third party material in this article are included in the article's Creative Commons license, unless indicated otherwise in a credit line to the material. If material is not included in the article's Creative Commons license and your intended use is not permitted by statutory regulation or exceeds the permitted use, you will need to obtain permission directly from the copyright holder. To view a copy of this license, visit http://creativecommons.org/licenses/by/4.0/. 
recently shown that defective intestinal barrier function caused by cell shedding in the terminal ileum predicts relapse of inflammatory bowel disease (IBD) in humans ${ }^{4}$. Defects in intestinal barrier function may also be involved in the pathogenesis of systemic conditions, such as metabolic endotoxaemia and sepsis ${ }^{5,6}$.

Several pro-inflammatory cytokines are present at increased concentrations in the circulation, intestinal lumen and lamina propria of the intestine during active IBD, infectious diarrhoea, coeliac disease and sepsis ${ }^{7-12}$. Tumour necrosis factor (TNF) is a well characterised cytokine that is produced in these conditions and is a key mediator of mucosal pathology $y^{3,13}$. Elevated TNF concentrations in excess of $63 \mathrm{ng} / \mathrm{g}$ stool have been documented in humans in response to Shigella flexneri infection $^{11}$. Anti-TNF therapies are also widely used clinically to ameliorate active Crohn's disease ${ }^{14}$.

We have recently shown that the administration of lipopolysaccharide (LPS) or its downstream effector, TNF, by intraperitoneal injection to mice results in a massive induction of epithelial apoptosis and cell shedding from the SI villus tip within $1.5 \mathrm{~h}^{4,15-17}$. This rapid onset of active caspase- 3 positively stained shedding cells subsequently resulted in villus atrophy and was accompanied by fluid effusion into the SI lumen and diarrhoea, but was largely diminished at $3 \mathrm{~h}$ post LPS injection ${ }^{17}$. However, increased efflux of FITC-dextran (FD4) from the intestinal lumen into the circulation following LPS treatment was observed at later time-points ${ }^{17}$, suggesting that defects in intestinal barrier function persist once cell shedding and apoptosis have subsided, until complete restitution of the epithelium has been achieved. The regenerative capacity of the intestinal epithelium is remarkable. Cell turnover in the epithelium is normally around 5 days with around 1400 cells shed from a single mouse villus tip per day ${ }^{18}$. We would therefore anticipate that barrier function could take up to 5 days to be restored following extensive epithelial cell loss by apoptosis and cell shedding once inflammatory stimuli such as TNF and interferon $\gamma$ (IFN $\gamma$ ) have been removed. Understanding the mechanisms underpinning intestinal epithelial cell protection from cytokine-mediated injury will enable the future development of therapeutics for several intestinal and systemic diseases.

The NF- $\mathrm{kB}$ family of transcription factors consists of 5

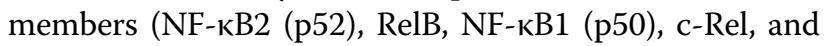
RelA (p65)) and regulate multiple cellular processes ${ }^{19}$. We have recently identified components of the alternative NF$\kappa \mathrm{B}$ signalling pathway that are important in modulating the susceptibility to IBD, colitis-associated cancer and intestinal epithelial apoptosis and cell shedding in mice. $N f \kappa b 2^{-1-}$ mice were resistant to dextran sulphate sodium (DSS)-induced colitis and azoxymethane/DSS-induced colonic adenoma formation ${ }^{20}$ and were also resistant to the induction of LPS and TNF-induced SI apoptosis and cell shedding in vivo ${ }^{16,17}$. Infection studies have also shown that $N f k b 2^{-1-}$ mice have a reduced ability to clear the gut helminth Trichuris muris ${ }^{21}$ and the gastric pathogen Helicobacter felis, the latter observation being associated with reduced gastric preneoplastic pathology owing to a defective immune respons $\mathrm{e}^{22}$. The complex in vivo nature of these studies to date, has made it impossible to determine the importance of each cellular compartment (e.g., immune or epithelial) in regulating the intestinal response to damage-inducing stimuli. The majority of studies characterising alternative pathway NF$\kappa B$ signalling are also focused on immune cell mechanisms which may be differentially regulated in other cellular compartments. Therefore, we have now generated a SI enteroid model to assess the interactions between the intestinal epithelium and specific immune cell derived cytokines that are known to be elevated in intestinal and systemic disease, and to determine the importance of epithelial cell-specific alternative pathway NF- $\mathrm{kB}$ signalling in regulating the epithelial cell response to cytokineinduced pathology.

Dendritic cells are present throughout the lamina propria of the gut and contribute to innate and adaptive immunity thus regulating gut homoeostasis. Direct bidirectional interaction of dendritic cells with the epithelium is believed to maintain the dendritic cell population in a tolerogenic state and this protects the intestinal epithelium from inappropriate immune cellderived attack ${ }^{23}$. Whilst dendritic cells are the most potent antigen presenting cell population, they are also activated by pro-inflammatory cytokines or oxidative stress and are conditioned by their surrounding microenvironment ${ }^{24}$. Cytokines and chemokines released by dendritic cells along with other lamina propria immune cell populations can modulate intestinal barrier integrity, intestinal cell proliferation and cell death ${ }^{25}$. Our previous whole mouse global $N f_{k} b 2$ knockout studies ${ }^{17,20,22}$ have limited our ability to dissect the importance of alternative pathway NF- $\mathrm{B}$ signalling within epithelial and immune compartments in regulating the susceptibility of the intestinal epithelium to cytokine-induced injury. We therefore generated a bone marrow-derived dendritic cell (BMDC) reconstituted intestinal organoid model to assess the role of NF- $\mathrm{kB} 2$ in regulating intestinal epithelial cell-specific responses to secreted factors from BMDCs.

We hypothesise that $N f_{\kappa} b 2$ activation within intestinal epithelial cells sensitises them to the induction of apoptosis by pro-inflammatory cytokines that are upregulated in intestinal tissues and systemically during active intestinal disease and bacteraemia. We have therefore explored whether Nfkb2 inhibition within intestinal epithelia could be a potential therapeutic approach to ameliorating 
inflammation-associated intestinal disease using a novel reconstituted intestinal organoid co-culture model.

\section{Results}

Regional differences are observed in enteroid morphology

The small intestine (SI) displays regional differences in structure and function. The expression of several genes is altered along the cephalocaudal axis in vivo and this region-specific identity has previously been shown to be maintained in enteroid culture ${ }^{26,27}$. We therefore generated organoids from proximal, middle and distal $2 \mathrm{~cm}$ segments of murine SI from female C57BL/6J mice and assessed their morphological changes over 10 consecutive passages. Enteroids derived from the three distinct areas displayed morphological differences between regions and these did not alter following repeated passage. At days 3-5 post passage, proximal SI derived enteroids had the longest $(116 \pm 3 \mu \mathrm{m})$ and most abundant crypt domains (7.22 \pm 0.26 /enteroid) with smooth surfaces and a polarised Paneth cell distribution at the base of crypts, whilst the distal SI derived enteroids had a smaller number of crypt domains (4.04 \pm 1.02 /enteroid) which were shorter $(89 \pm 4 \mu \mathrm{m})$ and displayed a more rounded and rugged appearance with a less polarised Paneth cell distribution with these differentiated cells also being found further along the crypt axes (Fig. 1a). Middle SI derived enteroids had $5.22 \pm 1.90$ crypts per enteroid that were $91 \pm 11 \mu \mathrm{m}$ in length and showed the greatest degree of variation in crypt length and number and Paneth cell distribution, suggestive of an intermediate phenotype.

\section{TNF induces enteroid apoptosis and Paneth cell depletion,} but does not modulate enteroid proliferation

We previously identified subtle regional differences in the murine SI in vivo in response to intraperitoneally administered LPS. A greater extent of villus blunting was observed in the proximal SI compared with other SI regions ${ }^{17}$. We therefore investigated the apoptotic and proliferative responses to a downstream effector of LPS, TNF, in region-specific enteroid cultures compared to histological sections taken from the same regions of the intestine in vivo. Baseline amounts of apoptosis in both untreated SI and in enteroid culture regardless of region were low, however, a small increase in the percentage of active caspase- 3 positive cells was observed along the cephalocaudal axis in both systems (Fig. 1b-e). As expected, intraperitoneal injection of $0.33 \mathrm{mg} / \mathrm{kg}$ TNFinduced a significant increase in apoptotic cells at the villus tip after $1.5 \mathrm{~h}$ (Fig. 1b, d), whilst $100 \mathrm{ng} / \mathrm{ml}$ TNFinduced apoptosis to a similar extent in enteroids after $24 \mathrm{~h}$ (Fig. 1c, e).

Paneth cells are important for maintaining the intestinal stem cell niche, although the exact mechanisms by which this cell population supports the proliferative capacity and number of neighbouring crypt base columnar cells remain unknown. However, TNF has previously been shown to induce Paneth cell dysfunction in vivo ${ }^{28}$, and this could potentially impact on the regenerative capacity of the intestinal epithelium. We therefore investigated whether TNF modulated Paneth cell number in SI tissue and in enteroids as this could impact on crypt-villus growth dynamics. TNF administration caused a more marked and significant reduction in Paneth cell number in the proximal SI and proximal tissue-derived enteroids compared with other SI regions (Fig. 2a, b) suggesting that TNF may modulate the intestinal stem cell niche in different ways within the proximal SI compared with other intestinal regions. Olfactomedin-4 (Olfm4) is a putative active stem cell marker that also labels early transit-amplifying cells. Olfm4 expression was not altered in SI tissues treated with TNF but due to the distribution differences in cellular location of Olfm4 observed in enteroids, Olfm4 expressing cells were not possible to quantify reliably (Supplementary Fig. 1). We also wanted to test whether another abundant secretory cell type was affected by the addition of TNF and found that the goblet cell population in different regions of SI and in enteroids was not modulated by the addition of TNF (Supplementary Fig. 2).

Proliferation in the SI in vivo occurs within the transitamplifying region of the crypt; a distinctly different part of the crypt-villus axis to the villus tip, which is the region most susceptible to apoptosis in response to systemically administered TNF. As we have shown that TNF causes apoptosis and Paneth cell depletion at the dose and timepoints tested in mice in vivo and in enteroids, we wanted to determine whether this treatment had any impact on intestinal proliferation. Proliferation indicated by Ki67 positively stained nuclei was identified in the crypt domains of whole SI tissue and enteroids. TNF treatment had no significant effect on cellular proliferation either in vivo or in enteroid culture, in any of the three SI regions at the doses and time points that showed an increased apoptotic response and Paneth cell depletion (Fig. 2c, d).

\section{Apoptosis and resultant cell shedding into the lumen correlate positively with enteroid circularity}

Villus shortening occurs in vivo as a result of an increased rate of apoptosis and cell shedding from the villus tip with no compensatory increase in cellular proliferation (Figs. 1 and 2) ${ }^{17}$. Similar atrophy also occurs in enteroid cultures. As a result of cell loss by apoptosis with no accompanying change in cellular proliferation (Figs. 1 and 2), cells are shed into the enteroid lumen and these are not immediately replaced. Junctional complexes hold remaining epithelial cells together whilst there is likely pressure from the build-up of apoptotic bodies within enteroid lumens. This results in the enteroids gaining a 




more spherical morphology. We have quantified this enteroid 'rounding' in terms of circularity from 2dimensional images and have validated this as a reproducible and robust method of cell death analysis in enteroids following cytokine treatment (materials and methods). Three days post passage, untreated enteroids had circularity values of $\sim 0.35$, however, circularity increased to $\sim 0.6548 \mathrm{~h}$ following $100 \mathrm{ng} / \mathrm{mL}$ TNF and $\sim 0.9$ with conditioned media from LPS-activated BMDCs in C57BL/6J proximal tissue-derived enteroids (Fig. 3a). We validated the reproducibility of the scoring method by assessment of intra-scorer variability where the same person (L.J.) scored images from the same 12 enteroids with a range of different circularities 1 week apart (Fig. $3 \mathrm{~b})$, and inter-scorer variability where two different blinded scorers (L.J. and C.D.) assessed enteroid circularity from the same 12 enteroid images (Fig. 3c). We also determined that there was no correlation between 


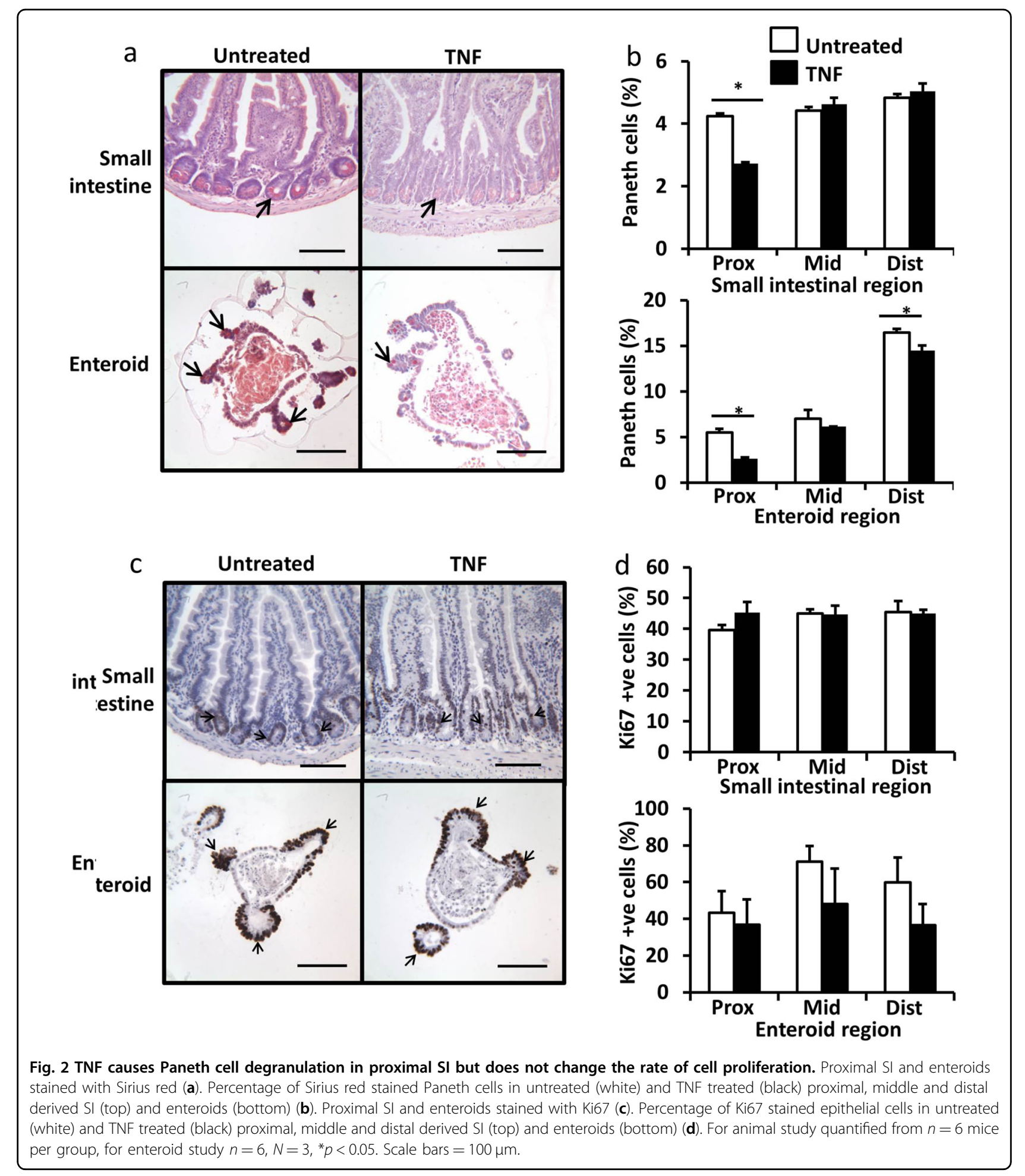

enteroid area and circularity as long as organoids were above a critical size at the start of each experiment, which equated to 3 days post passage or an initial baseline circularity value $<0.4$ (Fig. $3 \mathrm{~d}$ ).

To further validate the assessment of circularity as a reliable indicator of cytokine-induced cell death in proximal tissue-derived enteroids, we observed a good positive correlation $\left(R^{2}=0.6\right)$ between enteroid circularity and active caspase- 3 immunohistochemical staining (Fig. 3e). TNF activates apoptosis via caspase- 8 and active caspase- 8 is observed at the villus tip during intestinal cell shedding ${ }^{29}$. Active caspase- 8 was not detected at later 


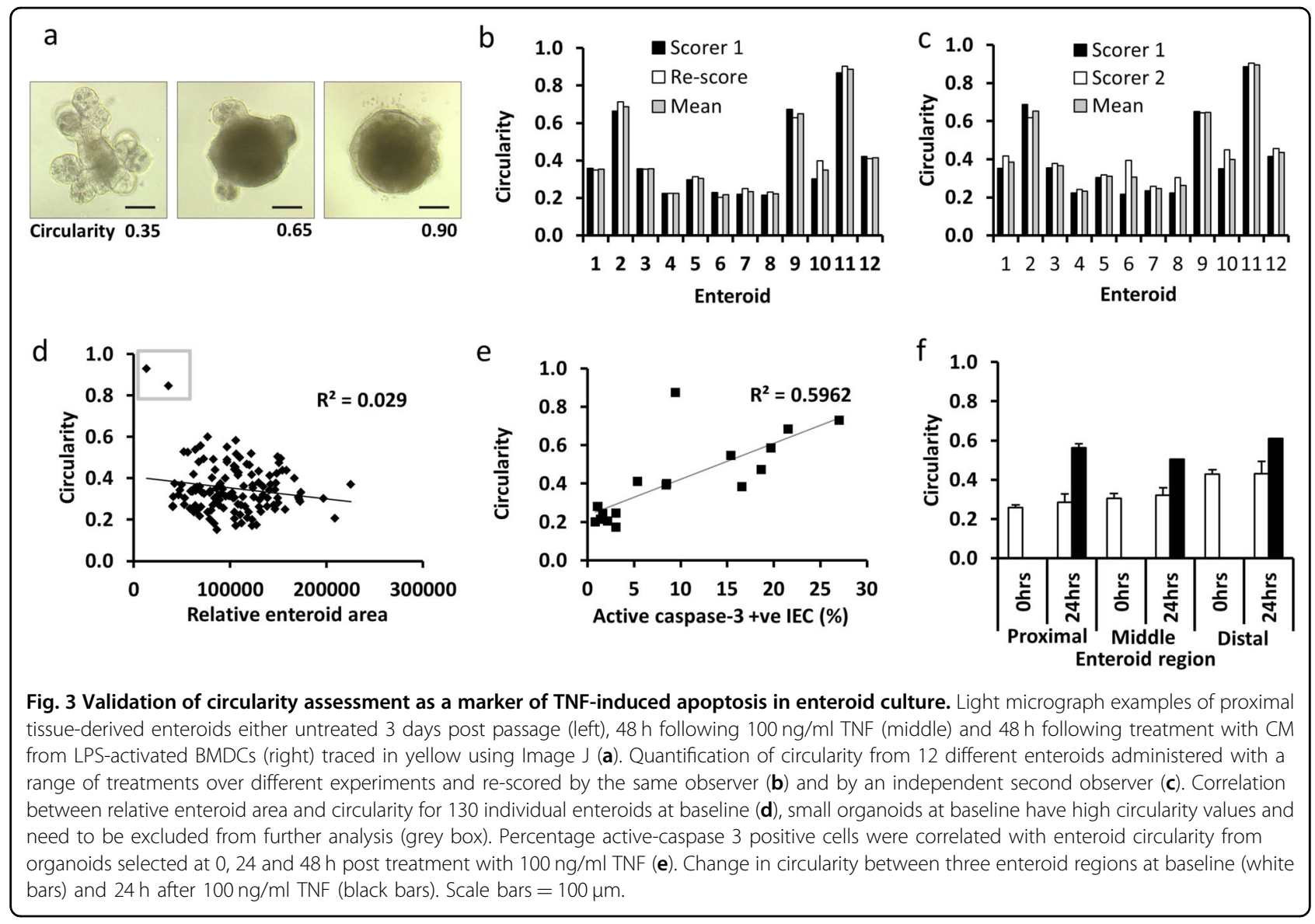

time-points during enteroid studies (Supplementary Fig. 3 ). As enteroids derived from the middle or distal segments of the SI displayed a more rounded appearance in culture at baseline (Fig. 1a), they also showed a blunted response to TNF in terms of increased circularity compared with proximal tissue-derived enteroids (Fig. 3f). Future studies should therefore consider the use of the circularity method for determining enteroid response to injury, however this method is not suitable for comparing responses between enteroid regions. We therefore used proximal tissue-derived enteroids for the remainder of this study.

\section{Alternative NF-KB pathway signalling in small intestinal epithelial cells regulates the response to cytokine-induced injury}

We have previously shown that $N f_{\kappa} b 2^{-1-}$ mice are resistant to TNF-induced villus tip apoptosis and cell shedding compared with their wild-type C57BL/6J counterparts ${ }^{17}$. However, the mechanism responsible for this resistance could not be determined during these in vivo studies as it was impossible to quantify the contribution of specific cellular compartments such as epithelial cells or immune cells. Using proximal tissue-derived enteroids, we have now assessed the importance of NFкB2 signalling specifically in the epithelial cell compartment in response to TNF and IFN $\gamma$ stimulation. Both TNF and IFN $\gamma$ caused C57BL/6J and $N f_{\kappa} b 1^{-1-}$ mousederived enteroids to undergo morphological changes and round up over a 24-h period, with further noticeable rounding after $48 \mathrm{~h}$ (Fig. $4 \mathrm{a}, \mathrm{b})$. This response was however blunted in $N f_{k} b 2^{-/-}$enteroids (Fig. 4c). A dosedependent increase in circularity was observed in C57BL/ 6J and $N f_{k} b 1^{-/-}$enteroids from 1 to $1000 \mathrm{ng} / \mathrm{mL}$ TNF and from 0.1 to $100 \mathrm{ng} / \mathrm{mL} \mathrm{IFN \gamma}$ at both $24-$ and 48 -h post treatment, however, no such increase was observed in $N f_{k} b 2^{-1-}$ enteroids (Fig. $5 \mathrm{a}-\mathrm{f}$ ) suggesting that alternative pathway NF- $\mathrm{B}$ activation is an important modulator of the intestinal epithelial cell response to injury.

LPS-activated bone marrow-derived dendritic cells induce enteroid rounding in a co-culture model and this is inhibited by epithelial NF-кB2 deletion

LPS administration to mice in vivo resulted in abundant villus tip apoptosis and cell shedding $1.5 \mathrm{~h}$ following intraperitoneal injection. We have previously shown Tnfr $1^{-1-}$ and Tlr $4^{-1-}$ mice to be resistant to LPS-induced cell shedding, suggesting that LPS acts via 


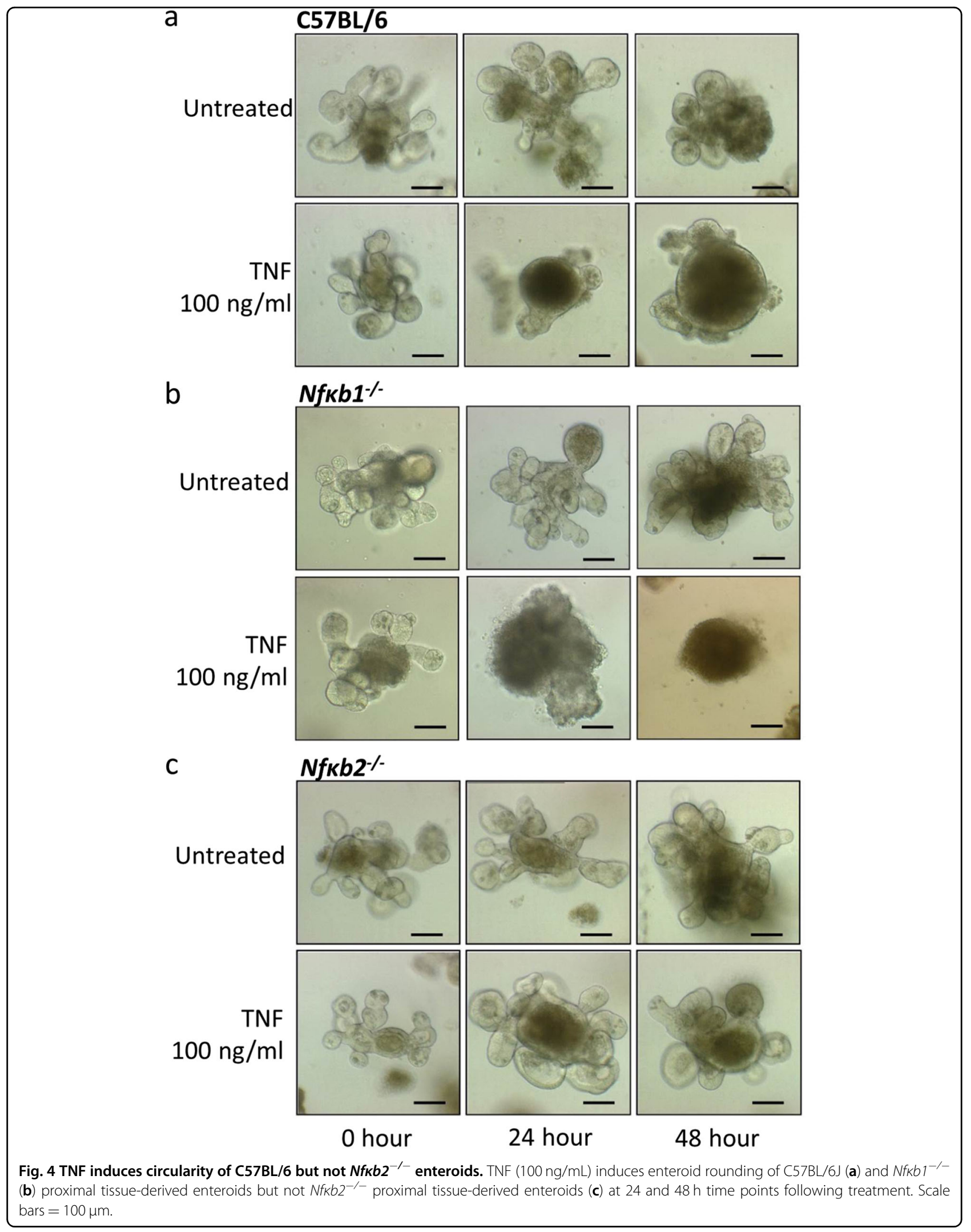




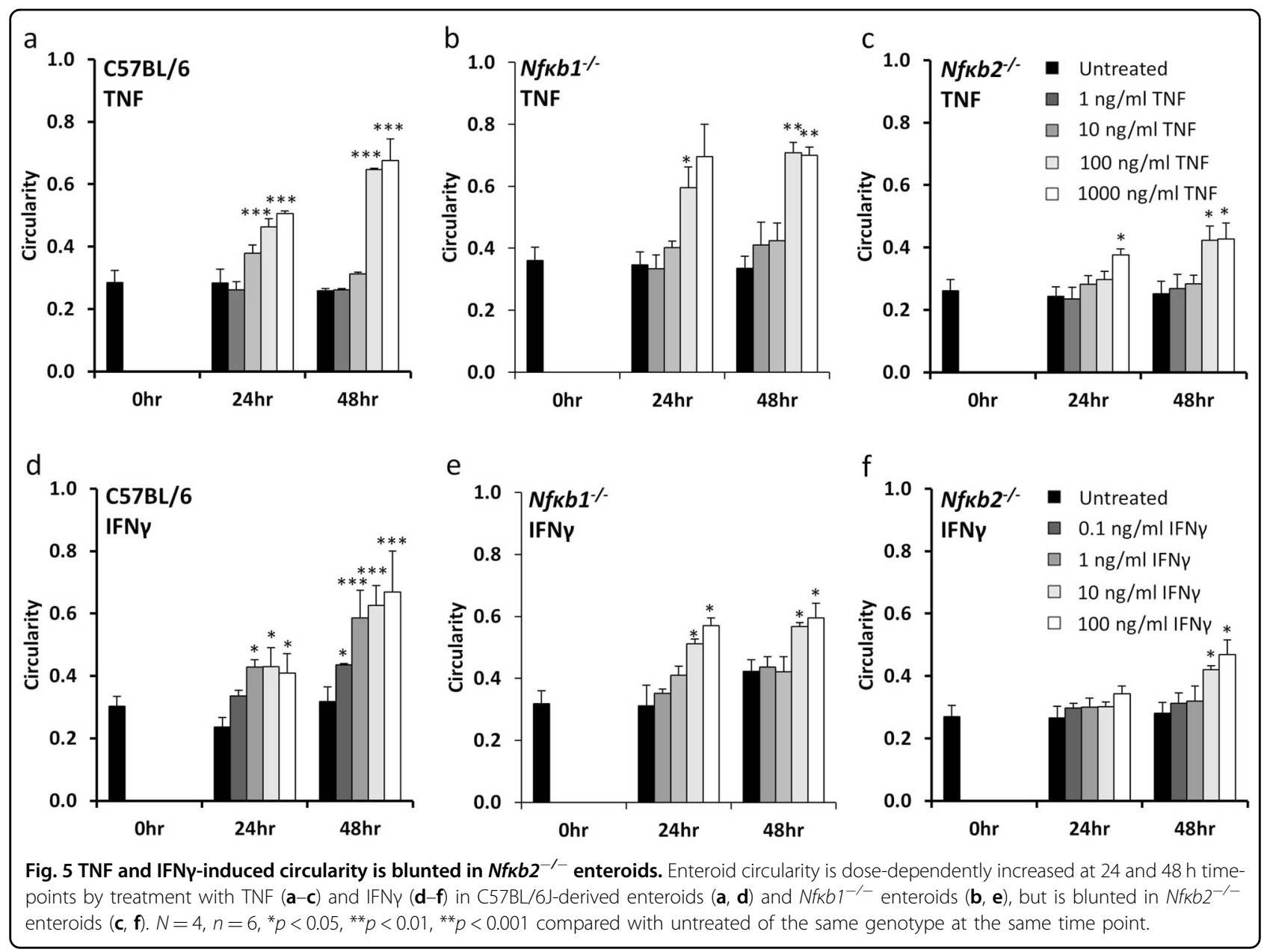

TLR4 on immune cells to induce ligands that bind the TNFR1 on epithelial cells to initiate the shedding process ${ }^{17}$. Dendritic cells have important functions in the innate and adaptive immune systems and are activated by LPS. We therefore co-cultured C57BL/6J-derived BMDCs with C57BL/6J, $N f \kappa b 1^{-/-}$or $N f \kappa b 2^{-/-}$enteroids and tested BMDCs that had or had not been activated by LPS. The direct application of $1 \mu \mathrm{g} / \mathrm{mL}$ LPS or unstimulated BMDCs had no morphological effect on any enteroid genotype (Fig. 6a) and we did not observe any significant differences in enteroid circularity following treatment (Fig. 6b-d). Interestingly, co-culture of C57BL/6J and $N f k_{k} b 1^{-1-}$ enteroids with LPS-activated BMDCs resulted in enteroid rounding (Fig. 6a) and a significant increase in enteroid circularity at both 24and 48 -h post treatment (Fig. $6 \mathrm{~b}, \mathrm{c}$ ). However, $N f_{\kappa} b 2^{-/-}$ enteroids were resistant to rounding and showed no significant increase in circularity in co-culture with LPSactivated BMDCs at 24 or $48 \mathrm{~h}$ (Fig. 6a, d). This suggests that NF- $\mathrm{kB} 2$ signalling specifically in intestinal epithelial cells is modulated by the interaction with dendritic cells.
Secreted factors from LPS-activated bone marrow-derived dendritic cells are responsible for organoid injury

We sought to determine whether the direct action of BMDC secreted factors on epithelial cells were responsible for the observed increase in C57BL/6J-derived enteroid circularity following stimulation with LPS. Conditioned media from unstimulated and LPSstimulated BMDCs was placed onto C57BL/6J enteroids. Conditioned media from BMDCs did not induce an increase in enteroid circularity, however, conditioned media from LPS-activated BMDCs induced a significant increase in enteroid circularity at both $24-$ and 48 -h post treatment (Fig. 7a, b). We assessed the cytokines that had been secreted by BMDCs into this conditioned media following stimulation with LPS and determined that several pro-inflammatory cytokines were upregulated. Initial analysis suggested that the amounts of IL-2, IL-4, IL-5 and IL-23 produced were minimal or below the lower limit of detection, hence these cytokines were not considered in subsequent experiments. TNF was upregulated in response to LPS and reached maximal concentrations of 

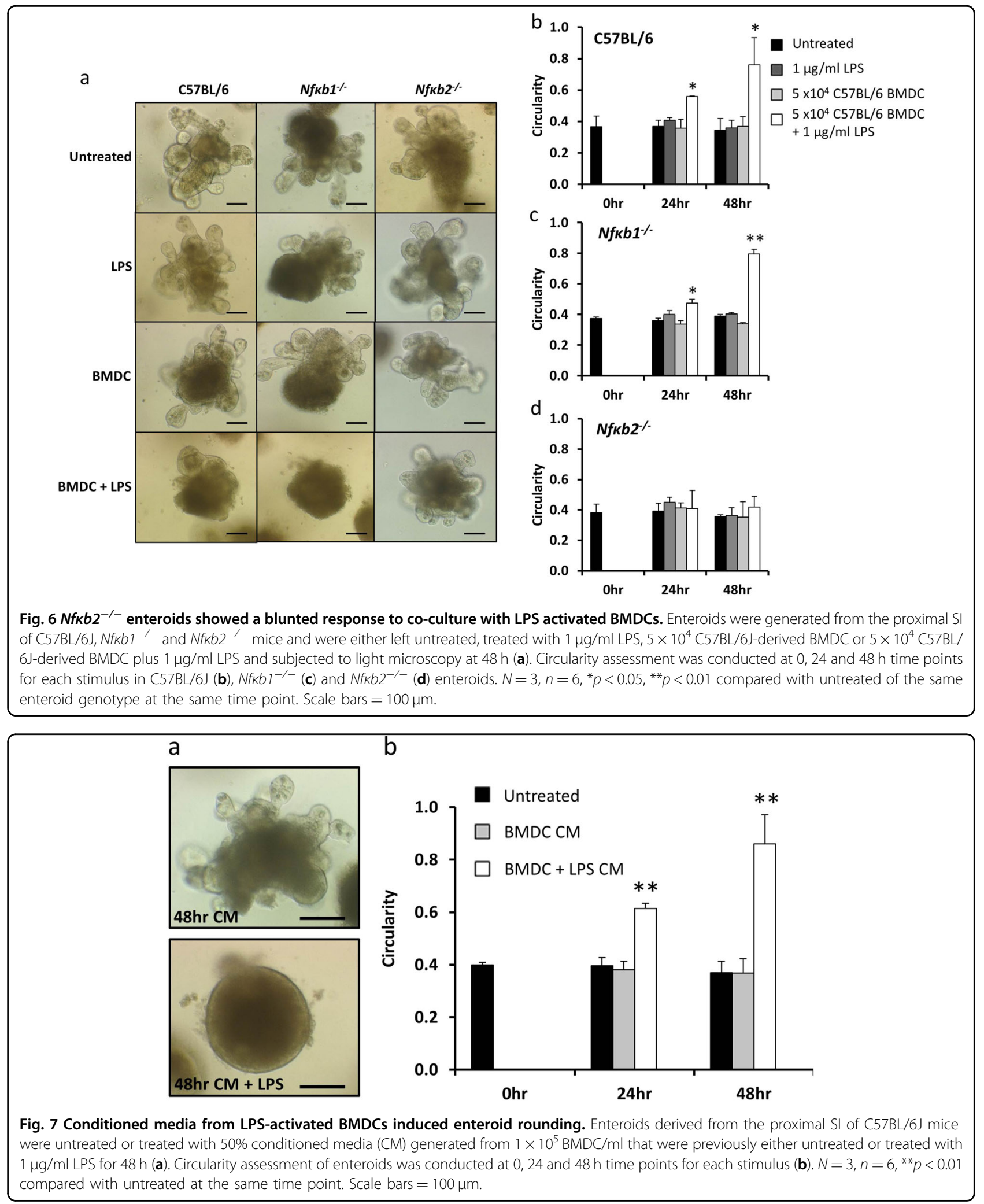

around $1 \mathrm{ng} / \mathrm{mL}$ (Table 1). However, this concentration was not high enough to stimulate an increase in enteroid circularity alone (Figs. 4 and 5). Significant increases in
IL-6, IL-1 $\beta$, IL-17A and IL-15 were also detected in the media of LPS-activated BMDCs in co-culture with enteroids and these cytokines may therefore have 
Table 1 Cytokines produced by C57BL/6J proximal tissue-derived enteroids, enteroids in co-culture with unstimulated BMDCs and enteroids in co-culture with LPS $(1 \mu \mathrm{g} / \mathrm{ml})$ activated BMDCs.

\begin{tabular}{llcc}
\hline Cytokine & $\begin{array}{l}\text { Enteroid } \\
(\mathbf{p g} / \mathbf{m l})\end{array}$ & $\begin{array}{l}\text { Enteroid }+ \\
\text { BMDC (pg/ml) }\end{array}$ & $\begin{array}{l}\text { Enteroid + BMDC }+ \\
\text { LPS (pg/ml) }\end{array}$ \\
\hline IFN-y & $0.09 \pm 0.02$ & $0.05 \pm 0.02$ & ND \\
IL-10 & ND & $98.42 \pm 43.94$ & $51.18 \pm 30.89$ \\
IL-12 p70 & ND & $2.35 \pm 2.03$ & $320.85 \pm 94.57$ \\
IL-15 & $0.40 \pm 0.40$ & $10.81 \pm 6.37$ & $186.74 \pm 42.01^{*}$ \\
IL-17A & $0.01 \pm 0.01$ & $0.01 \pm 0.01$ & $1.51 \pm 0.28^{*}$ \\
IL-13 & ND & $0.61 \pm 0.28$ & $42.90 \pm 5.36^{*}$ \\
IL-33 & $0.60 \pm 0.15$ & $0.30 \pm 0.19$ & $4.97 \pm 2.92$ \\
IL-6 & $2.14 \pm 0.82$ & $31.61 \pm 14.81$ & $43967.35 \pm 4220.72^{*}$ \\
KC/GRO & $4.16 \pm 0.55$ & $5.52 \pm 1.96$ & $103.43 \pm 30.89$ \\
TNF & $2.29 \pm 0.51$ & $180.37 \pm 78.95$ & $806.86 \pm 335.23$ \\
\hline
\end{tabular}

Analysis from three independent experiments in duplicate, data presented \pm SEM. $N D=$ not detected. ${ }^{*} p<0.05$ between enteroid + BMDC + LPS and enteroid + BMDC groups

contributed to the observed increase in enteroid circularity. Secreted factors from dendritic cells are therefore able to modulate intestinal epithelial cell response to injury, which can be modelled in an enteroid system.

\section{Discussion}

Recent advances have enabled long-term three-dimensional culture of the mammalian SI epithelium ${ }^{30-32}$. However, many published studies have not considered the differences that exist in structure and function between different anatomical regions of the SI in vivo. The duodenum, jejunum and ileum are each specialised compartments which have distinct absorptive and secretory functions ${ }^{26,33-36}$. Previous studies in human and mouse enteroids have indicated that cultures retain their regionspecific identities following multiple passage, in the absence of mesenchymal cell signalling or other niche factors $^{27}$. For instance, the Gata 4 transcription factor is highly expressed in all epithelial cells of the duodenum, but shows greatly reduced expression further down the cephalocaudal axis, with no expression being detected in the terminal ileum ${ }^{26,27}$. Gata 4 has been shown to activate jejunal-specific genes such as $C l n d 2$ (encoding Claudin-2 protein) but represses ileal-specific genes such as Slc10a2 (solute carrier family 10, member 2) and Fabp6 (encoding fatty acid binding protein 6$)^{26,37}$. Overexpression of Claudin-2 has been associated with inflammatory bowel disease and coeliac disease ${ }^{38}$ and is also upregulated in the intestine within $1 \mathrm{~h}$ of experimentally induced sepsis ${ }^{39}$. Regional differences in susceptibility occur for each of these pathologies and this is likely to result from differences in gene expression and environmental cues.

We therefore considered SI location when establishing enteroids and have identified variations in growth dynamics between three different regions of the intestine at baseline and following TNF treatment. These differences were also observed in parallel in vivo studies. In particular, Paneth cell numbers were increased from proximal to distal SI in vivo and in enteroid culture and we noted a greater amount of Paneth cell degranulation in the proximal SI both in vitro and in vivo in response to TNF. Previous studies have indicated that there is a greater degree of LPS-induced SI shedding and villus atrophy in the proximal SI compared with other regions ${ }^{17}$, however, loss of Paneth cell function within the crypt domains may also contribute to an alteration in the SI stem cell niche, thus perturbing cell production and dynamics further along the crypt-villus axis. We did not observe differences in the proliferative compartment along the length of the intestine by Ki67 immunohistochemistry (IHC), however, a large population of cells are labelled by Ki67 in both systems and more subtle differences in the proliferative compartment may be apparent by using more sensitive detection methods. It was difficult to compare the overall growth rate of organoids between the three regions, however, distal tissuederived enteroids were more difficult to establish than those from other regions of the SI and displayed morphological differences that were maintained for greater than ten passages. Therefore, future studies should consider that following successive culture, proximal tissuederived enteroids may outcompete distal tissue-derived derived enteroids in a mixed population.

One of the major cytokines that is responsible for the development of IBD and which is a major contributor to the cytokine storm and multiple organ dysfunction (including the gut epithelium) that occurs in sepsis is $\mathrm{TNF}^{4,7,17}$. The direct impact of TNF on enteroid morphology was striking. Over a period of $48 \mathrm{~h}$, enteroids adopted a rounded appearance and many became almost spherical. This altered morphology was a likely result of increased apoptosis and cell shedding into the enteroid lumen. Analogous to bubble formation, a spherical appearance may result from the line of least tension across tight and adherens junctions with pressure build up from shed cellular debris and secretions within the enteroid lumen. The observed change in enteroid morphology was assessed by measuring circularity and this correlated linearly with active caspase- 3 expression, thus validating the technique as a method for analysing cell death in this setting. This technique is much quicker than other similarly reported methods such as the assessment of branching morphogenesis by assessing the number and length of crypt buds ${ }^{40}$. The circularity assessment method 
is therefore useful for determining dose and time points for further investigations of a mechanistic nature, but requires optimisation and validation for each culture media formulation and damaging stimulus. The mechanisms of rounding are unlikely to be the same for all stimuli, however, this assay is particularly useful to determining the morphological impact on intestinal epithelia. Active caspase- 3 is relatively stable compared with other caspases and has a half-life of $8-11 \mathrm{~h}^{41}$ and was detectable following TNF treatment for the entire timecourse of our experiments. We also assessed the expression of active caspase- 8 and whilst this was observed at the villus tip at early time-points as in previous studies ${ }^{29}$, this caspase is much less stable, cleaved components p43 having a half-life of $63 \pm 5 \mathrm{~min}$ and p18 having a much shorter half-life of $7 \pm 1 \mathrm{~min}^{42}$ and was not detected over the whole time-course of our experiments in enteroids, thus active caspase- 8 activity did not positively correlate with enteroid circularity.

Upregulation of NF-kB2 mRNA has been observed in colitis-associated dysplasia and carcinoma ${ }^{43}$. Our previous in vivo studies have also demonstrated that $N f_{\kappa} b 2^{-1-}$ mice are less susceptible to the induction of experimental colitis, colitis-associated cancer and LPS-induced SI cell shedding ${ }^{17,20}$. However, the role of the intestinal epithelium in contributing to this protection was previously unclear as $N f f_{k} b 2^{-/-}$mice have a perturbed immune system that could have also contributed to the altered phenotype $^{44}$. We have now demonstrated that $N f_{\kappa} b 2^{-/-}$ proximal SI epithelium is resistant to TNF and IFNY induced pathology in enteroids in the absence of contributions from the other cellular compartments that are present in vivo. This suggests that NF- $\mathrm{KB} 2$ signalling is an important determinant of intestinal epithelial cell homoeostasis. A range of concentrations of cytokines was tested in our experiments and alterations in circularity were noted at concentrations greater than $10 \mathrm{ng} / \mathrm{ml}$ TNF and $0.1 \mathrm{ng} / \mathrm{ml}$ IFN $\gamma$. These concentrations are higher than those detected in the circulation and tissues of human patients, however, faecal concentrations have been noted between 1 and $100 \mathrm{ng} / \mathrm{g}$ and are in line with the concentrations used in this study ${ }^{11}$. Murine TNF is a known regulator of both classical and alternative pathway NF- $\mathrm{BB}$ signalling and is likely to mediate its effects via TNFR1 and 2 ligation. However, it was surprising to also observe a decreased sensitivity to IFN $\gamma$ in $N f \kappa b 2^{-1-}$ enteroids, though this is likely to be via an indirect mechanism resulting in the production of an NF- $\mathrm{KB} 2$ activator such as $\mathrm{BAFF}^{45}$. In addition to TNF and IFN $\gamma$, many cytokines contribute to the development of intestinal pathologies. We generated a co-culture model incorporating enteroids and BMDCs and showed that these cell populations could co-exist without any change to enteroid morphology. However, upon activation by LPS, BMDCs induced significant morphological changes in wild-type enteroids, whereas $N f_{k} b 2^{-1-}$ enteroids were seemingly protected. Cytokines generated in the model included TNF and IFNY but both were produced at much lower concentrations than were required alone to induce circularity changes in enteroids and were similar to those observed in patients with pathology. This suggests that other secreted factors may also act synergistically with TNF and IFN $\gamma$ to induce enteroid rounding in this setting. IL- 6 was produced in abundance and could therefore contribute to modulating epithelial cell dynamics. An in-depth investigation of the BMDC secretome and assessment of cytokine combinations is now warranted to determine how the intestinal mucosal microenvironment regulates epithelial cell dynamics in combination with genetic susceptibility that may modulate the threshold for cytokines to induce a pathological response.

These data suggest that the NF-kB2 signalling pathway, specifically in intestinal epithelial cells, may be targetable to ameliorate intestinal and systemic inflammation.

\section{Materials and methods \\ Animals}

Ten- to 12-week old wild-type C57BL/6J (Charles River, Margate, UK) and $N f_{\kappa} B 1^{-1-}$ and $N f \kappa b 2^{-/-}$mice ${ }^{44,46}$, bred on the $\mathrm{C} 57 \mathrm{BL} / 6 \mathrm{~J}$ genetic background were maintained at the University of Liverpool under a 12:12 $\mathrm{h}$ light dark cycle and fed a standard pelleted diet. All mice were euthanized by cervical dislocation with ethical approval under UK Home Office legislation (Animals Scientific Procedures Act 1986) and local ethical approval.

\section{TNF treatment}

Six female C57BL/6J mice were administered $0.33 \mathrm{mg} /$ $\mathrm{kg}$ murine recombinant TNF (Peprotech Ltd., London, UK) by intraperitoneal injection and euthanized $1.5 \mathrm{~h}$ later. Six untreated female C57BL/6J were used as controls, mice were randomly allocated to two groups prior to treatment. SIs were excised, flushed with phosphatebuffered saline (PBS) pH 7.4 and fixed in $10 \% \mathrm{v} / \mathrm{v}$ neutral buffered formalin maintaining orientation using previously described methods ${ }^{47}$.

\section{Tissue preparation and scoring}

Formalin-fixed SI was divided up into proximal, middle and distal thirds and bundled using methods previously described $^{47-49}$. Bundles were routinely processed, embedded in paraffin wax in the transverse orientation and $4 \mu \mathrm{m}$ thick tissue sections were stained with haematoxylin and eosin (H\&E), direct red 80 (both from Sigma, Dorset, UK) or prepared for IHC.

For the quantification of apoptotic and proliferative intestinal epithelial cells in immunostained tissue sections, individual epithelial cells were counted from the 
base of the crypt to the mid-point of the villus tip in 20 well-orientated hemi crypt-hemi villi at 400× magnification from 6 mice per group (group sizes of 6 were estimated to be needed from previous studies to provide appropriate statistical power and the scorer was blinded to animal identifier at the time of analysis) ${ }^{17}$. For the quantification of apoptotic and proliferative intestinal epithelial cells in immunostained enteroid sections, individual cells were counted in the total circumference of 6 whole enteroids from at least 3 independent replicate studies at $400 \times$ magnification. Labelled cells were counted if in continuation with the epithelial monolayer. Intestinal epithelial cells were categorised according to the following criteria:

1. Normal if there was no or weak non-specific brown staining.

2. Apoptotic/mitotic if there was defined positive staining confined to cytoplasmic or nuclear borders.

\section{IHC and histochemical staining}

Tissue and enteroid sections were deparaffinised and rehydrated. Standard histochemical techniques were used for H\&E, direct red 80 (Sirius red) and alcian blue staining, the latter two being counterstained with haematoxylin to identify nuclei for quantification. Immunohistochemically stained slides were subjected to $1 \% \mathrm{v} / \mathrm{v}$ hydrogen peroxide in methanol to block endogenous peroxidases, followed by heat-mediated antigen retrieval in $0.01 \mathrm{M}$ citrate acid buffer $(\mathrm{pH} 6)$ and then blocked with $1 \% \mathrm{w} / \mathrm{v}$ bovine serum albumin (Sigma, Dorset, UK). Rabbit polyclonal primary antibodies against active caspase-3 (AF835; R\&D Systems, Abingdon, UK), Ki67 (AB15580; Abcam, Cambridge, UK) and olfactomedin-4 (\#39141; Cell Signalling Technology, UK) were applied at $2.5,2$ and $0.25 \mu \mathrm{g} / \mathrm{mL}$, respectively to sections for $2 \mathrm{~h}$ and visualisation was completed using an anti-rabbit Envision kit (Dako, Cambridge, UK) polymer followed by peroxidase substrate 3,3'-diaminobenzidine (DAB) (Sigma, Dorset, UK) using the manufacturer's instructions. Sections were counterstained with haematoxylin.

\section{Bone marrow-derived dendritic cell extraction and culture}

Femurs and tibias were harvested from male C57BL/6J wild-type mice and were mechanically cleaned to remove any surrounding tissues. Bones were transferred into $70 \%$ $\mathrm{v} / \mathrm{v}$ ethanol for $2 \mathrm{~min}$ to sterilise their outsides. Bones were clipped at the top and bottom and a $24 \mathrm{G}$ needle was used to flush out the bone marrow through a $40 \mu \mathrm{m}$ cell sieve. Red blood cells were lysed, cells were quantified and resuspended in dendritic cell differentiation solution (RPMI containing GM-CSF $(20 \mathrm{ng} / \mathrm{ml})$, $\beta$-mercaptoethanol $(50 \mu \mathrm{M}), 10 \% \mathrm{w} / \mathrm{v}$ FCS, $1 \% \mathrm{w} / \mathrm{v}$ glutamine, $1 \% \mathrm{w} / \mathrm{v}$ penicillin/streptomycin; all from Sigma,
Dorset, UK) in $10 \mathrm{~cm}$ petri dishes at a concentration of $2.5 \times 10^{5} / \mathrm{mL}$ and plated at $20 \mathrm{~mL} /$ plate. Fresh media was added to dishes 4 days following and non-adherent BMDCs were used in experiments 7 days following isolation and differentiation.

BMDC conditioned media was generated by plating BMDCs in complete RPMI media as described above at concentrations of $1 \times 10^{3}, 1 \times 10^{4}, 1 \times 10^{5} \mathrm{BMDC} / \mathrm{mL}$. BMDCs were removed from culture media by centrifugation after $48 \mathrm{~h}$ and supernatants were immediately used in enteroid assays.

\section{Enteroid culture}

Extraction and culture of SI crypts was carried out as previously described ${ }^{32}$ with modifications as indicated below. Male 10-week-old C57BL/6J mice were killed and the SI was rapidly removed and flushed with ice-cold PBS. The most proximal, middle and most distal $2 \mathrm{~cm}$ segments were isolated separately, opened out longitudinally, cut into $1 \mathrm{~cm}$ lengths and placed into PBS. Intestinal segments were washed 10 times in ice-cold PBS and incubated with ice-cold chelation buffer $(20 \mathrm{~mL}$ of $2 \mathrm{mM}$ EDTA in PBS) at $4{ }^{\circ} \mathrm{C}$ with constant gentle agitation for $30 \mathrm{~min}$. Chelation buffer was then replaced with $20 \mathrm{~mL}$ shaking buffer ( $43.3 \mathrm{mM}$ sucrose, $59.4 \mathrm{mM}$ sorbitol; Sigma, Dorset, UK) and shaken gently for $30 \mathrm{~s}$ to remove the majority of villi. Shaking buffer was replaced with fresh shaking buffer $(20 \mathrm{~mL})$ and crypts were then detached from the basal membrane by vigorous shaking by hand for $2 \mathrm{~min}$. The crypt-enriched supernatant was filtered through a $70 \mu \mathrm{m}$ cell strainer (BD Biosciences Heidelberg, Germany) and quantified using microscopy. The filtered crypts were centrifuged at $200 \times g \quad\left(4{ }^{\circ} \mathrm{C}, 10 \mathrm{~min}\right)$ and resuspended at 10,000 crypts $/ \mathrm{mL}$ in Matrigel (BD Biosciences) containing growth factors; epidermal growth factor $(50 \mathrm{ng} /$ $\mathrm{mL})$, R-spondin-1 $(500 \mathrm{ng} / \mathrm{mL})$ and Noggin $(100 \mathrm{ng} / \mathrm{mL})$ (R\&D Systems). Approximately, 500 crypts in $50 \mu \mathrm{L}$ Matrigel were seeded per well of a pre-warmed 24-well flat-bottomed plate and incubated at $37^{\circ} \mathrm{C}$ for $10 \mathrm{~min}$ to polymerise the Matrigel. Finally, $500 \mu \mathrm{L}$ of minigut culture medium was added to each well (advanced DMEM/F12, $1 \%$ L-glutamine, $1 \%$ Pen/Strep, $10 \mathrm{mM}$ HEPES, 10 mM N2 supplement, 50 mM B27 supplement (Invitrogen)) and crypts were cultured at $37{ }^{\circ} \mathrm{C}$ in a $95 \%$ air, $5 \% \mathrm{CO}_{2}$ atmosphere.

All enteroids were passaged at least once prior to use in an experiment. Passage was carried out weekly at a 1:4 split ratio. Media was removed and enteroids in Matrigel were resuspended in PBS and mechanically dissociated into crypts by passing through a $27 \mathrm{G}$ needle. Dissociated enteroids were then centrifuged at $200 \times g$ $\left(4{ }^{\circ} \mathrm{C}, 10 \mathrm{~min}\right)$, resuspended in fresh Matrigel containing growth factors and plated as above. Culture medium was 
changed and replaced with new media containing growth factors every 4 days.

\section{Enteroid treatments}

C57BL/6J, $N f \kappa b 1^{-/-}$and $N F \kappa b 2^{-/-}$enteroids (passage $2-12)$ were treated 3 days following passage by adding murine recombinant TNF $(1-1000 \mathrm{ng} / \mathrm{mL}$; PeproTech Ltd., London, UK), IFN $\gamma$ (01-100 ng/mL; Peprotech Ltd.), C57BL/6J BMDCs $\left(5 \times 10^{4} /\right.$ well $)$ with or without $1 \mu \mathrm{g} / \mathrm{mL}$ LPS diluted in $50 \%$ minigut media (containing $2 \times$ growth factors) and 50\% complete BMDC media or conditioned media from BMDCs. High power images of 3 enteroids/ well, conducted in duplicate were taken at 0,24 and $48 \mathrm{~h}$ for circularity measurements and enteroids were harvested for histological analysis as described below.

\section{Enteroid histology}

Following treatment with TNF, media was removed and replaced with $500 \mu \mathrm{L}$ cell recovery solution (Corning, New York, USA) and agitated at $4{ }^{\circ} \mathrm{C}$ until the Matrigel was dispersed. Enteroids were then fixed in $2 \% \mathrm{v} / \mathrm{v}$ neutral buffered formalin. After 1-h fixation enteroids were centrifuged at $100 \times g$ for $1 \mathrm{~min}$. The supernatant was discarded and $80-120 \mu \mathrm{l}$ of pre-warmed Histogel ${ }^{\mathrm{TM}}$ (Thermo Scientific, Massachusetts, USA) was mixed carefully with enteroids and transferred directly onto a section of Micropore $^{\mathrm{TM}}$ surgical tape forming a button when set. Sections of tape with Histogel were transferred into embedding cassettes and routinely processed and embedded in paraffin wax. Sections of Histogel containing enteroids $(4 \mu \mathrm{m}$ thick) were stained with $\mathrm{H} \& \mathrm{E}$, direct red 80 , or prepared for IHC as described above.

\section{Enteroid circularity assessment}

Bright field microscopy was used to monitor enteroid development from crypt isolation and throughout subsequent passages and treatments. Images were taken using an Axiovert 25 microscope (Zeiss) and a 20× objective lens with a Hitachi HV-C20A camera. Enteroid morphology was assessed throughout experiments using 2D bright field images and a software-based formula for calculating circularity: $4 \pi$ (area)/perimeter ${ }^{2}$. Two-dimensional images of enteroids were traced (three representative images per well conducted in duplicate) and analysis was conducted by recording enteroid area, perimeter and circularity using the analyse and measure function in Image software ${ }^{50}$. Circularity values are possible between 0 and 1 , with a score of 1 indicating a perfect circle. The circularity scoring method was adapted for enteroids from a previously reported system in mammary epithelial cultures ${ }^{51}$.

\section{Cytokine analysis}

Media was removed from enteroids and enteroids in coculture with BMDCs with and without LPS treatment $48 \mathrm{~h}$ post culture and centrifuged to remove debris. Samples were immediately frozen at $-80^{\circ} \mathrm{C}$ until further analysis and defrosted only once for further usage. Samples were run in duplicate and a multiplexing capable analysis platform selected for simultaneous cytokine profiling (QuickPlex SQ 120, Meso Scale Discovery, USA). The Uplex mouse cytokine assay system was employed for the analysis of a panel of markers as per manufacturer's instructions. Cytokines analysed were: IFN $\gamma$, TNF, KC/ GRO, IL-1 $\beta$, IL-2, IL-4, IL-5, IL-6, IL-10, IL-12 p70, IL-15, IL-17, IL-23 and IL-33. Quantitative measurement of each marker was achieved using a four-parameter logistic regression method.

\section{Statistical analysis}

Student's $t$ tests were carried out on normally distributed data within proximal, middle and distal regions of the SI and for cytokine production between LPS-treated BMDCs and untreated BMDCs. All additional normally distributed data were analysed by one-way ANOVA versus control group followed by Holm-Sidak post hoc analysis. Statistical tests that achieved $p<0.05$ were considered significant.

\section{Acknowledgements \\ C.A.D. was supported by the Fiona Elizabeth Agnew Trust (FEAT), The Physiological Society, Epistem Ltd. and by a tenure-track fellowship from the University of Liverpool. D.M.P., B.J.C. and M.D.B. were supported by the European Community's Seventh Framework Programme (FP7/2007-2013) under grant agreement No. 305564 (SysmedIBD). C.A.D. and B.J.C. were additionally supported by a Wellcome Trust 4-year PhD studentship to L.T. (105231/Z/14/Z) in Cellular \& Molecular Physiology at the University of Liverpool. We thank the Biomedical Services Unit, University of Liverpool and the Technology Directorate for maintenance of mice used in this study. We are also grateful to Bristol-Myers Squibb for providing Nfkb2 $2^{-1-}$ mice and David Baltimore for providing $\mathrm{Nfkb}^{-1-}$ mice.}

\section{Author details}

${ }^{1}$ Gastroenterology Research Unit, Department of Cellular and Molecular Physiology, Institute of Translational Medicine, University of Liverpool, Liverpool, UK. ${ }^{2}$ Institute of Immunology and Immunotherapy, College of Medical and Dental Sciences, University of Birmingham, Birmingham, UK. ${ }^{3}$ Department of Clinical and Molecular Pharmacology, Institute of Translational Medicine, University of Liverpool, Liverpool, UK. 'Biotechnology Group, Oswaldo Cruz Foundation, Ceara, Brazil. ${ }^{5}$ Pathobiology and Population Sciences, Royal Veterinary College, Hawkshead Lane, North Mymms, Hatfield, Hertfordshire, UK

Conflict of interest

The authors declare that they have no conflict of interest.

\section{Publisher's note}

Springer Nature remains neutral with regard to jurisdictional claims in published maps and institutional affiliations.

Supplementary Information accompanies this paper at (https://doi.org/ 10.1038/s41419-019-2129-5).

Received: 9 July 2019 Revised: 23 October 2019 Accepted: 11 November 2019

Published online: 26 November 2019 


\section{References}

1. Potten, C. S., Booth, C. \& Pritchard, D. M. The intestinal epithelial stem cell: the mucosal governor. Int J. Exp. Pathol. 78, 219-243 (1997).

2. Williams, J. M. et al. Epithelial cell shedding and barrier function: a matter of life and death at the small intestinal villus tip. Vet. Pathol. 52, 445-455 (2015).

3. Watson, A. J., Duckworth, C. A., Guan, Y. \& Montrose, M. H. Mechanisms of epithelial cell shedding in the mammalian intestine and maintenance of barrier function. Ann. N. Y. Acad. Sci. 1165, 135-142 (2009).

4. Kiesslich, R. et al. Local barrier dysfunction identified by confocal laser endomicroscopy predicts relapse in inflammatory bowel disease. Gut 61, 1146-1153 (2012)

5. Cani, P. D. et al. Changes in gut microbiota control metabolic endotoxemiainduced inflammation in high-fat diet-induced obesity and diabetes in mice. Diabetes 57, 1470-1481 (2008).

6. Piton, G., Manzon, C., Cypriani, B., Carbonnel, F. \& Capellier, G. Acute intestinal failure in critically ill patients: is plasma citrulline the right marker? Intensive Care Med 37, 911-917 (2011).

7. Soufli, I., Toumi, R., Rafa, H. \& Touil-Boukoffa, C. Overview of cytokines and nitric oxide involvement in immuno-pathogenesis of inflammatory bowel diseases. World J. Gastrointest. Pharm. Ther. 7, 353-360 (2016).

8. Korolkova, O. Y., Myers, J. N., Pellom, S. T., Wang, L. \& M'Koma, A. E. Characterization of serum cytokine profile in predominantly colonic inflammatory bowel disease to delineate ulcerative and Crohn's colitides. Clin. Med. Insights Gastroenterol. 8, 29-44 (2015).

9. Braegger, C. P., Nicholls, S., Murch, S. H., Stephens, S. \& MacDonald, T. T. Tumour necrosis factor alpha in stool as a marker of intestinal inflammation. Lancet 339, 89-91 (1992).

10. Murch, S. H., Braegger, C. P., Walker-Smith, J. A. \& MacDonald, T. T. Location of tumour necrosis factor alpha by immunohistochemistry in chronic inflammatory bowel disease. Gut 34, 1705-1709 (1993).

11. Nicholls, S., Stephens, S., Braegger, C. P., Walker-Smith, J. A. \& MacDonald, T. T. Cytokines in stools of children with inflammatory bowel disease or infective diarrhoea. J. Clin. Pathol. 46, 757-760 (1993).

12. Bjorck, S., Lindehammer, S. R., Fex, M. \& Agardh, D. Serum cytokine pattern in young children with screening detected coeliac disease. Clin. Exp. Immunol. 179, 230-235 (2015)

13. Piguet, P. F., Vesin, C., Donati, Y. \& Barazzone, C. TNF-induced enterocyte apoptosis and detachment in mice: induction of caspases and prevention by a caspase inhibitor, ZVAD-fmk. Lab. Invest. 79, 495-500 (1999).

14. Bandzar, S., Gupta, S. \& Platt, M. O. Crohn's disease: a review of treatment options and current research. Cell Immunol. 286, 45-52 (2013).

15. Duckworth, C. A. \& Watson, A. J. Analysis of epithelial cell shedding and gaps in the intestinal epithelium. Methods Mol. Biol. 763, 105-114 (2011).

16. Marchiando, A. M. et al. The epithelial barrier is maintained by in vivo tight junction expansion during pathologic intestinal epithelial shedding. Gastroenterology 140, 1208-1218 (2011). e1201-1202.

17. Williams, J. M. et al. A mouse model of pathological small intestinal epithelial cell apoptosis and shedding induced by systemic administration of lipopolysaccharide. Dis. Models Mech. 6, 1388-1399 (2013).

18. Potten, C. S. \& Loeffler, M. Stem cells: attributes, cycles, spirals, pitfalls and uncertainties. Lessons Crypt. Dev. 110, 1001-1020 (1990).

19. Merga, Y. J. et al. Importance of the alternative NF-kappaB activation pathway in inflammation-associated gastrointestinal carcinogenesis. Am. J. Physiol. Gastrointest. Liver Physiol. 310, G1081-G1090 (2016).

20. Burkitt, M. D. et al. NF-kappaB1, NF-kappaB2 and c-Rel differentially regulate susceptibility to colitis-associated adenoma development in C57BL/6 mice. J. Pathol. 236, 326-336 (2015).

21. Artis, D. et al. Differential requirement for NF-kappa B family members in control of helminth infection and intestinal inflammation. J. Immunol. 169 4481-4487 (2002)

22. Burkitt, M. D. et al. Signaling mediated by the NF-kappaB sub-units NF-kappaB1, NF-kappaB2 and c-Rel differentially regulate Helicobacter felis-induced gastric carcinogenesis in C57BL/6 mice. Oncogene 32, 5563-5573 (2013).

23. Gross, M., Salame, T. M. \& Jung, S. Guardians of the gut-murine intestinal macrophages and dendritic cells. Front. Immunol. 6, 254 (2015).

24. Bernardo, D., Chaparro, M. \& Gisbert, J. P. Human intestinal dendritic cells in inflammatory bowel diseases. Mol. Nutr. Food Res. 62, e1700931 (2018).
25. Andrews, C., McLean, M. H. \& Durum, S. K. Cytokine tuning of intestinal epithelial function. Front. Immunol. 9, 1270 (2018).

26. Bosse, T. et al. Gata4 is essential for the maintenance of jejunal-ileal identities in the adult mouse small intestine. Mol. Cell. Biol. 26, 9060-9070 (2006).

27. Middendorp, S. et al. Adult stem cells in the small intestine are intrinsically programmed with their location-specific function. Stem Cells 32, 1083-1091 (2014).

28. Van Hauwermeiren, F. et al. TNFR1-induced lethal inflammation is mediated by goblet and Paneth cell dysfunction. Mucosal Immunol. 8, 828-840 (2015).

29. Gunther, C. et al. Caspase-8 controls the gut response to microbial challenges by Tnf-alpha-dependent and independent pathways. Gut 64, 601-610 (2015).

30. Derricott, $H$. et al. Developing a 3D intestinal epithelium model for livestock species. Cell Tissue Res. 375, 409-424 (2018).

31. Sato, T. \& Clevers, H. Primary mouse small intestinal epithelial cell cultures Methods Mol. Biol. 945, 319-328 (2013).

32. Sato, T. \& Clevers, H. Growing self-organizing mini-guts from a single intestinal stem cell: mechanism and applications. Science 340, 1190-1194 (2013).

33. Gordon, J. I., Schmidt, G. H. \& Roth, K. A. Studies of intestinal stem cells using normal, chimeric, and transgenic mice. FASEB J. 6, 3039-3050 (1992).

34. Gulec, S., Anderson, G. J. \& Collins, J. F. Mechanistic and regulatory aspects of intestinal iron absorption. Am. J. Physiol. Gastrointest. Liver Physiol. 307, G397-G409 (2014).

35. Kapadia, C. R. \& Donaldson, R. M. Jr. Disorders of cobalamin (vitamin B12) absorption and transport. Annu. Rev. Med. 36, 93-110 (1985).

36. Tappenden, K. A. Pathophysiology of short bowel syndrome: considerations of resected and residual anatomy. JPEN J. Parenter. Enter. Nutr. 38(1 Suppl), 14S-22S (2014).

37. Lepage, D. et al. Gata4 is critical to maintain gut barrier function and mucosal integrity following epithelial injury. Sci. Rep. 6, 36776 (2016).

38. $\mathrm{Hu}, \mathrm{C}$. A. et al. Autophagy and tight junction proteins in the intestine and intestinal diseases. Anim. Nutr. 1, 123-127 (2015).

39. Yoseph, B. P. et al. Mechanisms of intestinal barrier dysfunction in sepsis. Shock 46, 52-59 (2016).

40. Fuller, M. K. et al. Intestinal crypts reproducibly expand in culture. J. Surg. Res. 178, 48-54 (2012)

41. Walsh, J. G., Logue, S. E., Luthi, A. U. \& Martin, S. J. Caspase-1 promiscuity is counterbalanced by rapid inactivation of processed enzyme. J. Biol. Chem. 286, 32513-32524 (2011).

42. Kallenberger, S. M. et al. Intra- and interdimeric caspase-8 self-cleavage controls strength and timing of CD95-induced apoptosis. Sci. Signal. 7, ra23 (2014).

43. Gillespie, E. et al. Plasminogen activator inhibitor-1 is increased in colonic epithelial cells from patients with colitis-associated cancer. J. Crohn's Colitis 7, 403-411 (2013).

44. Caamano, J. H. et al. Nuclear factor (NF)-kappa B2 (p100/p52) is required for normal splenic microarchitecture and B cell-mediated immune responses. J. Exp. Med. 187, 185-196 (1998).

45. Woo, S. J. et al. Induction of BAFF expression by IFN-gamma via JAK/STAT signaling pathways in human intestinal epithelial cells. J. Leukoc. Biol. 93, 363-368 (2013).

46. Sha, W. C., Liou, H. C., Tuomanen, E. I. \& Baltimore, D. Targeted disruption of the p50 subunit of NF-kappa B leads to multifocal defects in immune responses. Cell 80, 321-330 (1995).

47. Williams, J. M., Duckworth, C. A., Vowell, K., Burkitt, M. D. \& Pritchard, D. M. Intestinal preparation techniques for histological analysis in the mouse. Curr. Protoc. Mouse Biol. 6, 148-168 (2016).

48. Duckworth, C. A. \& Pritchard, D. M. Suppression of apoptosis, crypt hyperplasia, and altered differentiation in the colonic epithelia of bak-null mice. Gastroenterology 136, 943-952 (2009).

49. Duckworth, C. A. et al. Progastrin-induced secretion of insulin-like growth factor 2 from colonic myofibroblasts stimulates colonic epithelial proliferation in mice. Gastroenterology 145, 197-208 e193 (2013).

50. Schneider, C. A., Rasband, W. S. \& Eliceiri, K. W. NIH Image to ImageJ: 25 years of image analysis. Nat. Methods 9, 671-675 (2012).

51. Plichta, K. A., Mathers, J. L., Gestl, S. A., Glick, A. B. \& Gunther, E. J. Basal but not luminal mammary epithelial cells require PI3K/mTOR signaling for Ras-driven overgrowth. Cancer Res. 72, 5856-5866 (2012). 